\title{
A Study of Hybridization Understanding from Algorithmic to Conceptual - Is Algorithmic an End Point for Students?
}

Gianna J. Manchester, ${ }^{\dagger}$ Julia E. Winter, ${ }^{\dagger}$ Sean P. Hickey, ${ }^{\ddagger}$ Sarah E. Wegwerth ${ }^{*} \dagger$

$\dagger$ Alchemie Solutions Inc, Troy, Michigan, 48083, United States

¥ Wayne State University, Detroit, Michigan, 48202, United States

\section{ABSTRACT}

Despite the call to remove hybridization theory from chemistry curricula, its teaching persists today in both general chemistry and organic chemistry courses. Because a major argument against hybridization is that students never develop a conceptual understanding, the research presented in this paper investigates the conceptual basis of the strategies students use to approach common hybridization assessment questions. Students' mental visualizations of hybrid orbitals were also examined. To accomplish this, the authors adapted a methodology developed by Niaz, which separated student reasoning into a series of models, to help determine a student's initial level of

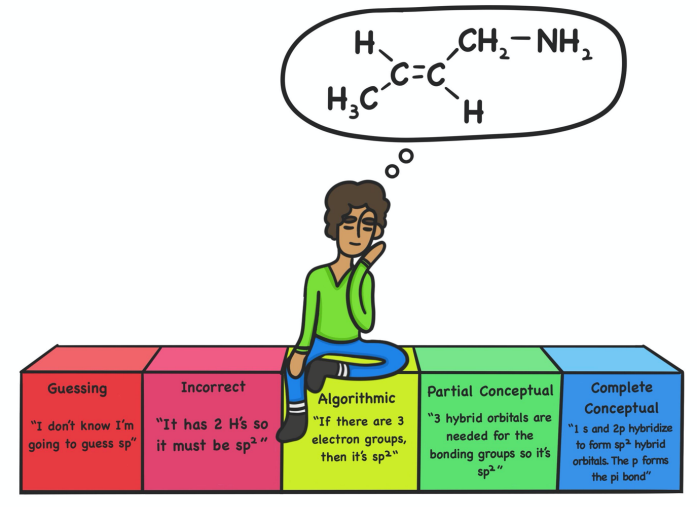
understanding and comprehension regarding hybridization problems. Niaz's research suggests that student understanding of a chemical concept lies on a continuum from algorithmic recall to conceptual understanding. Think-aloud interviews were conducted at three institutions with both general and organic chemistry students to examine thought processes with respect to solving a set of five hybridization questions. Additionally, students were asked to draw the orbitals of a $s p^{2}$-hybridized carbon atom and to indicate the orbital used for pi bonding. The student responses to the non-drawing questions were categorized into one of five models of thinking that ranged from guessing to algorithmic thinking to full, conceptual understanding of the question. Analysis of the models indicated that most students use algorithmic thinking to answer a question, even for conceptually based questions. Some students did attempt to apply conceptual thinking to augment or confirm the answer, but many only used algorithmic thinking for their answers. Examination of student drawings revealed that none of the participants could render and describe a complete and accurate image of all the orbitals for a trigonal carbon. Additionally, no correlation was found between ability to answer conceptual questions and accuracy of drawings indicating a weakness in integrating the symbolic and sub-microscopic domains.

KEYWORDS

First-Year Undergraduate / General, Second-Year Undergraduate, Chemical Education Research, Organic Chemistry, Misconceptions / Discrepant Events, Problem Solving / Decision Making, Covalent Bonding, Lewis Structures, Properties / Structure, Valence Bond Theory, VSEPR Theory

\section{INTRODUCTION}

In the Journal of Chemical Education, Grushnow posited that the continued teaching of hybridization

to chemistry students was unwarranted since it was a model that does not accurately represent bonding and could easily be eliminated with "little negative effect."1 Multiple chemical educators have argued 
against Grushnow's assertions. ${ }^{2-6}$ However, in both Grushnow's initial arguments and his detractors' rebuttals, only anecdotal evidence was given without providing meaningful chemical education research to support their arguments. Grushnow's assertion to remove hybridization, while a useful thoughtexercise, has not resulted in any meaningful change in the chemistry curriculum. General chemistry textbooks still include a detailed discussion of hybridization as part of the introduction to the theory of bonding. ${ }^{7-10}$ Additionally, a brief, yet highly conceptual overview of hybridization is included in most organic chemistry textbooks in the review of general chemistry topics. ${ }^{11-14}$ Based on a survey of textbooks, it is logical to assume that a fairly detailed, conceptual approach of hybridization is taught in general chemistry (and reinforced for carbon in organic chemistry) as a necessary component for understanding bonding theory.

Therefore, this paper does not continue the discussion (opinions) on whether to keep or remove hybridization from the chemistry curricula using anecdotal data; but, instead focuses on a scientific analysis of students' thinking when solving hybridization questions to determine the usefulness of the hybridization model. To assess student thinking, a question needed to be asked and analyzed. "Are students getting the correct answer to hybridization questions by rote memorization and algorithmic recall or do they understand, and can they answer hybridization questions on a deeper, conceptual level?" The initial methodology for this study, the creation of the parent codes and models, and analysis of students' reasoning on the continuum of learning are discussed in this paper. Also evaluated are student drawings of the orbitals of a $s p^{2}$-hybridized carbon.

\section{Correct Answers vs Conceptual Understanding}

Grunshow's anecdotal claims are partially supported by studies that have documented students' development of alternative conceptions regarding atomic and hybrid orbitals. ${ }^{1,15-18}$ Additionally, understanding of hybridization appears to be particularly difficult for novice students. ${ }^{19,20}$ The existence of these misconceptions and comprehension challenges suggest a need for a different pedagogical approach, because if the misconception is more than just a single fact, as will likely happen with flawed mental model, then conceptual change is required. ${ }^{21}$

Orbitals are abstract, meaning they are not something we can observe or experience. In fact, it should be a benefit for chemistry educators that students are not bringing in misconceptions based on 
experiences from everyday life. ${ }^{18}$ Conversely though, this means that these misconceptions are the result of instructional experiences, both inside and outside the classroom, and, problematically, become pedagogic impediments. ${ }^{16,18}$ Student misconceptions can be corrected and avoided in the future once they are identified. As a success example, Zoller used schemes for methane that depicted the orbitals and change in energy levels, to define atomic orbitals and show the progression to hybrid orbitals. ${ }^{17}$ Finally, we must remember from constructivism that knowledge is constructed in the mind of the learner. ${ }^{22}$ Therefore, we should not be disappointed by the need of students to iterate their knowledge structures as they gain conceptual understanding but rather see it as a natural progression of learning. ${ }^{23}$ An instructor's job is to design learning experiences and scaffold material at a level appropriate for our students and guide them as they build deeper understandings. ${ }^{24}$

What still remains unknown is a proven pedagogy that leads to strong conceptual understanding of hybridization. ${ }^{18}$ We propose that the problem is two-fold; one, the questions asked do not require conceptual knowledge and two, current models of orbitals do not meet student needs. Answering common assessment questions for other chemistry topics, such as manipulating gas law equations, mechanisms in organic chemistry, and student understanding of structure property relationships, have been shown to require little conceptual knowledge. ${ }^{25-31}$ Stowe and Cooper, highlight how this misleads students as (ref 27, p 1853):

Assessments convey a strong implicit message to students about what is really important in a course... If tests and quizzes are composed only of items that can be answered via recall, application of simple algorithms, or patter recognition, then students will spend their time reading and rereading flashcards of reagents and reactions rather than on understanding the underlying constructs.

Correspondingly, we postulate that common hybridization assessment questions naturally steer students to develop and use "if this, then that" algorithms, especially those questions derived from the VSEPR chart that is included in many textbook discussions of valence bond theory.

While these algorithms provide an accessible working definition for orbitals, they have no conceptual meaning. To gain meaning, the concept must be understood. ${ }^{18}$ Once the terms have meaning, then a contextual framework is available to generate meaningful mental images. However, due to the abstract nature of orbitals and the complicated mathematics used to describe their shapes, 
students need extensive guidance on how to visualize orbitals. Hence, our second proposed pedagogical weakness is the availability of manipulatable models of orbitals. Historically, orbitals are presented in textbooks, which are limited to static two-dimensional images. Beyond images, a search of the internet results in various videos that use animations and even balloons as an aide to teach hybridization. More recently, 3D printers have allowed instructors to produce physical models of hybrid orbitals and student surveys reveal that students find the tool useful. ${ }^{32,33}$ A deficiency of these representations though is that none of them allow students to independently manipulate and construct orbital models for exploration or assessment purposes.

\section{Research Goals and Questions}

The overarching goal, of our ongoing hybridization research project, is to investigate if a digital interactive model of atomic and hybrid orbitals, referred to here on as the hybridization explorer, can help students develop a stronger conceptual understanding of hybridization theory. To observe changes in students' working knowledge of hybridization, think-aloud interviews were conducted with undergraduate students in both general chemistry and organic chemistry. The interviews consisted of three parts. First, students were asked to think-aloud as they filled out a questionnaire that consisted of five hybridization questions. Upon answering a question, students were also prompted to rate their confidence levels.

By having students fill out the questionnaire in a think-aloud interview, we were able to probe their reasoning. ${ }^{34}$ However, we recognize that our observations alone may not provide an accurate assessment of their conceptual understanding, as this has been shown to be dependent on the modality of the task. ${ }^{35}$ For this study though, we specifically wanted to learn if student strategies are purely algorithmic in nature or to what extent they incorporate conceptual reasoning. The goal of this work was to use a grounded theory research design to identify the reasoning students use to answer standard hybridization questions from the first questionnaire. Our research questions were:

1. What are the primary strategies students use to solve hybridization type question and where along the continuum of algorithmic to conceptual reasoning do these strategies lie?

2. How complete are student mental visualizations of orbitals as compared with accepted models?

3. How well does completeness of depictions of orbitals correlate with ability to answer conceptually based questions? 


\section{Framework}

For this study, we needed a theoretical framework to delineate a method to gauge conceptual understanding. To fulfill this requirement, we adopted a theory proposed by Niaz, that the strategies used by students to solve problems evolve from algorithmic reasoning to a reliance on conceptgrounded rationales with stronger explanatory power. ${ }^{23,31} \mathrm{~A}$ core component of this framework is that algorithmic and conceptual explanations are not dichotomous, but rather two ends of a continuum. As students are first introduced to topics, the material may be simplified, and one way to do that is to use algorithms to show relationships. While development of an algorithm requires solid conceptual understanding, frequently, use of the algorithm does not require conceptual understanding. Consequentially, students are comfortable using these algorithms, making it a preferential strategy for problem-solving by novices. ${ }^{31}$ As conceptual understanding increases though, these strategies evolve to incorporate conceptual knowledge which provides stronger explanatory reasoning.

To develop this theory, Niaz constructed models of student reasoning along the continuum. ${ }^{31}$ These models were numbered so that Model 1 represented the strategies at the algorithmic end and the highest number model was primarily conceptual reasoning. In his work he looked at several different topics and generated models specific for each topic.

The idea that student models progress as understanding increases also mirrors the process by which scientific knowledge is built. ${ }^{31}$ For example, in studying gases, individual relationships are studied through Boyle's law, Charles' law, and Avogadro's law. This leads to the Ideal Gas Law, a simplified model for how gases behave. However, to model and describe real gases more accurately would require conceptual understanding of particle interactions which cannot be grasped from equations alone. Additionally, Taber inferred, from detailed case studies, that while studying a concept a student may construct multiple alternative frameworks before "suddenly" understanding the topic. ${ }^{18}$ It is these transitions that are the observable change of a learner's conceptual knowledge. Therefore, it is not to say that algorithmic based problems and abilities are unimportant but that they should be a starting point rather than an end goal, as is suggested by many current exam questions. 


\section{METHODS}

Participants and Settings

This study was determined to be exempt from IRB review by Sterling IRB prior to recruitment. To participate in the study, volunteers needed to be at least 18 years of age or older, be enrolled in or have completed college level first semester general chemistry or its equivalent (such as AP chemistry) within the last year or be currently enrolled in a subsequent chemistry course. Recruitment emails were sent to three chemistry instructors at three different Midwestern universities who then forwarded the email to students currently enrolled in their course during the fall 2019 semester. Two were large public PhD granting universities and the email was shared with students in first semester organic chemistry at one and general chemistry laboratory courses at the other. The third was a small private university and was sent to students in organic chemistry. Students were offered a $\$ 20$ gift card as compensation for one hour of their time. Volunteers were enrolled based on who signed up first resulting in a convenience sampling. ${ }^{36}$ Verbal consent was obtained prior to starting the interview. No demographic or personal information was collected. All data were stored and reported in this paper using pseudonyms.

Semi-structured think-aloud interviews were used to elicit participants' strategies in solving traditional hybridization questions and their thought process while using the explorer. ${ }^{34,36}$ The interviews consisted of three phases, a five question (Q1-Q5) pre-test referred to as a questionnaire, a review of hybridization using the explorer, and a post-test, which was a fresh copy of the first questionnaire. The focus of this paper is on phase one and participants' responses to the questionnaire. The questionnaire was designed to assess participants' capabilities, learn the strategies and reasoning behind their answers, and gain insight into their mental visual of orbitals. As questions themselves can elicit different problem solving approaches, the questions varied in the degree of algorithmic and conceptual reasoning necessary to answer the question. ${ }^{25,26}$

The questionnaire (Figure S1) was developed by the last author and validity was established by the other authors who are chemistry content experts and the professors who sent out the recruitment emails. All questions involved the structure shown in Figure 1. Additionally, questions 1-3 and 5 were variants of questions found in the ACS organic chemistry exam study guide 37 and thus are assumed to 
be representative of standard hybridization questions. A more detailed rationale for each question, as well as the inclusion of recording self-reported confidence levels, is given in the Supplementary Information.

Questionnaires were filled out using an iPad pro. Interviews were recorded using an iPhone, or a web cam and a microphone, to capture the audio and a visual of the screen the participant was working on. Screen recordings of the iPad were also captured.

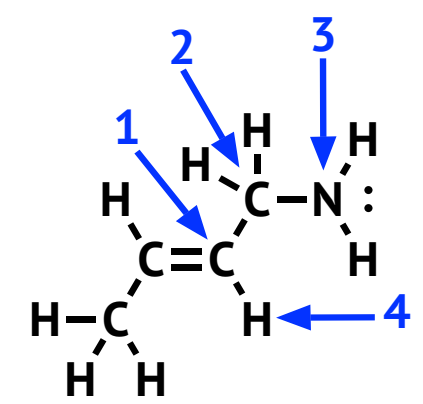

Figure 1. The Lewis structure, as presented, that was referred to by all questions on the questionnaire.

Data Analysis

All interviews were transcribed using the secure online service Temi. To ensure accurate

transcription, the transcripts were edited by members of the research team before being imported into the program Dedoose to aide with qualitative data analysis. Coding of the transcripts followed the practices of grounded theory and constant comparative analysis. ${ }^{34,36}$ Codes specific for each question, including Q4, were generated together by the first and last author using three transcripts. These codes identified the specific algorithm(s) and or concept(s), or lack thereof, used to develop each answer, and for Q4 described the features drawn. Then the first and last author separately coded four additional transcripts and found agreement of $100 \%$. The first author coded the remaining transcripts independently. Any new codes that were generated were discussed with the last author during daily debriefings to ensure agreement.

The initial child codes were then collapsed into parent codes (Table S1) that were the same for each question, except Q4 (discussed below), which categorized the basis of the strategy (i.e. a guess, deductive, algorithmic, conceptual). It should also be noted that for participants who invoked more than one strategy for a specific answer, more than one parent code may have been applied. 
From the parent codes, five models emerged to define the observed points along a continuum that describe the level of reasoning participants used. While the models were developed following Niaz's methodology, contrary to Niaz, we chose to encompass every strategy used by participants, whether correct or not, to gain a more complete picture. As a result, our continuum begins at an absence of reasoning (Model 1) and ends at proper conceptual reasoning (Model 5) as shown in Figure 2. To determine which model each answer belonged in, we considered both the parent code and the answer accuracy. For example, if the parent code algorithmic was present, then we also considered the correctness of the answer to determine if the response fit into Model 2 versus Model 3.

\begin{tabular}{|c|c|c|c|c|}
\hline $\begin{array}{l}\text { Model } 1 \\
\text { Participant } \\
\text { guesses with no } \\
\text { reasoning }\end{array}$ & $\begin{array}{c}\text { Model } 2 \\
\text { Uses invalid } \\
\text { reasoning or } \\
\text { uses reasoning } \\
\text { which leads to } \\
\text { an incorrect } \\
\text { answer }\end{array}$ & $\begin{array}{c}\text { Model } 3 \\
\text { Correctly uses } \\
\text { algorithmic } \\
\text { reasoning without } \\
\text { demonstrating any } \\
\text { conceptual } \\
\text { understanding }\end{array}$ & $\begin{array}{c}\text { Model } 4 \\
\text { Correctly answers } \\
\text { question and } \\
\text { demonstrates } \\
\text { partial conceptual } \\
\text { understanding }\end{array}$ & $\begin{array}{c}\text { Model } 5 \\
\text { Correctly answers } \\
\text { question and } \\
\text { demonstrates } \\
\text { complete } \\
\text { conceptual } \\
\text { understanding }\end{array}$ \\
\hline
\end{tabular}

Figure 2. The definitions of each of the five models of our continuum in order from guessing to complete conceptual understanding.

To test inter-rater reliability for the coding of Q1-Q3, and Q5, the third author independently coded one question from each participant (20\% of the data) after becoming familiar with the codebook (Table S2). To each question, he applied the applicable parent codes and model. The Cohen's Kappa value for application of the models was 0.73 indicating moderate agreement. ${ }^{38}$ Differences were then discussed and appropriate changes to the coding system were made. An additional 10\% of the data was coded by the third author and the Cohen's Kappa value was 0.85 indicating strong agreement. ${ }^{38}$

The goal of Q4 was to elicit what mental visualization students have of orbitals and therefore required a different coding scheme than the others. Q4 was split into three separate components: hybrid orbitals, atomic orbital, and pi bond. Each component was coded based on what participants said and drew while watching a replay of the interview. Child codes were developed simultaneously and followed the same process as the other questions. From the child codes, the last author developed and applied the parent codes which separately identified the completeness of each of the three components of the drawing. The second author verified the coding by independently analyzing $10 \%$ of the data. Agreement between the raters was minimal. ${ }^{38}$ The coding scheme was then discussed and 
revised by the first, second, and last authors. After developing a clear understanding of the revised codebook, the first author recoded all responses to Q4 and the second author independently coded an additional $10 \%$ of the data. $100 \%$ agreement was reached. The final coding schemes can be found in the Supplementary Information (Tables S3-S9).

\section{Limitations}

Even with the think-aloud format, some participants whose answer was classified as algorithmic may indeed have had a proper conceptual understanding but did not feel the need to invoke it to answer the question. This may be in part an artifact of the type of question asked (remembering that chosen were typical questions that are a mixture of algorithmic/conceptually based ones) but we also propose that this may be a consequence of current pedagogical practices. For Q4, some students did not name the orbitals drawn. In those cases, we assumed they were hybrid or atomic based on which one they more closely resembled.

\section{RESULTS AND DISCUSSION}

Although our goal was to evaluate student reasoning, a question itself can be characterized as algorithmic or conceptual in nature. Questions that can be answered by using a formula, or in this case a heuristic that does not require conceptual knowledge, are classified as algorithmic. Conversely, questions that require using chemical concepts to develop an answer are classified as conceptual. For this reason, we have organized this section to discuss the results of the questions in order of increasing level of conceptual basis rather than in numerical order. Thus, the order the questions (Q) are covered is Q1, Q3, Q5, Q2, and Q4. Finally, one last, but important caveat that the authors acknowledge is that some participants whose answer was classified as algorithmic may indeed have a complete conceptual understanding and did not feel the need to invoke it to answer the question. This is in part an artifact of the type of question asked (remembering that chosen were typical questions that are a mixture of algorithmic/conceptually based ones) but we also propose that this may be a consequence of current pedagogical practices.

Question 1: What is the hybridization of atoms C-1, C-2?

We classified Q1 as algorithmic since much of the hybridization curriculum leads students to count the number of electron groups then refer to a table (which in turn is memorized) to find the 
corresponding hybridization. This question proved to be the easiest question for participants with 24 using an algorithm to answer both parts correctly. The most common strategy $(n=23$, where $n$ is the number of instances the strategy was observed), as seen in Table 1, was counting the number of electron groups around each carbon which produced a satisfactory answer all but four times. Three of those four participants counted the double bond as two electron groups and the other incorrectly recalled the chart. The next most common $(n=7)$ algorithmic strategy participants used was to relate the bond types to the hybridization, recognizing that a carbon with all single bonds signifies $s p^{3}$ and a double bond signifies $s p^{2}$-hybridization. The 20 participants who successfully used one of these algorithms without further accurate conceptual support were placed in Model 3. Consequently, participants who answered incorrectly using algorithmic reasoning were placed into Model 2. Harold, for example, recognized the bond types and tried to make a correlation by saying: "they look like just single bonds and single to me is like putting a one here. So that's my reasoning, it's just $s p^{1}$. I guess my reasoning for the two [in $s p^{2}$ ] was because there's a double bond."

Table 1. A summary of the parent codes and associated child codes observed for $Q 1$, as well as the frequency each child code was observed and number of times it contributed to generating a correct answer and incorrect answer.

\begin{tabular}{ccccc} 
Parent Code & Child Code & $\begin{array}{c}\text { Number of } \\
\text { Times } \\
\text { Observed (n) }\end{array}$ & $\begin{array}{c}\text { Reasoning Led } \\
\text { to Correct } \\
\text { Answer }\end{array}$ & $\begin{array}{c}\text { Reasoning Led } \\
\text { to Incorrect } \\
\text { Answer }\end{array}$ \\
& Number of electron groups & 23 & 19 & 4 \\
& Bond Type & 7 & 5 & 2 \\
\hline Conceptual & Count Number of H's & 1 & 0 & 1 \\
\hline Other Concepts & Defining Orbitals for Bond & 6 & 5 & 1 \\
\hline
\end{tabular}

Natalia, like Harold, was also placed in Model 2, but unlike Harold, she was the only participant that did not use an algorithmic approach to Q1. Instead, she tried to "visualize" the orbitals and explain how they overlapped to form the double bond of C-2: "you have the two $p$ orbitals in the dumbbell shape, and then you have the regular $s$ orbital overlap, so it would just be sp." Although her reasoning relied on conceptual reasoning, it was ultimately deemed insufficient because of its lack of explanatory power to develop a sound answer. Additionally, the absence of an algorithm suggests that 
she has not yet grasped the introductory material which is why her answer, and others like it, were placed into Model 2.

The five participants who used the concept of orbitals to support their algorithmic strategy were successful in identifying each atom's hybridization. Georgia had the most in depth explanation by recognizing: "for C-1 there are three different electron groups and so there have to be three different, uh, hybrid orbitals cause the double bond only counts as one sigma bond." Since these conceptual responses lacked additional discussion about how the $s$ and $p$ orbitals combine to form the hybrid orbitals or how one $p$ orbital on $\mathrm{C}-1$ is used to form the pi bond, they were all placed in Model 4 and no one was in Model 5.

Overall, Question 1 was the question participants performed the best, likely because it is algorithmic in nature, which is also why the vast majority of participants were in Model 3 and only seven were in Model 2 (Figure 3). The fact that no one guessed without reasoning, Model 1, is indicative of the pervasiveness of the algorithmic strategies taught to students. Unsurprisingly, based on reports that students are more comfortable using algorithms than conceptual knowledge, ${ }^{31} \mathrm{Q} 1$ was also the question in which participants were most confident with 23 of them indicating that they were confident or very confident in their answer.

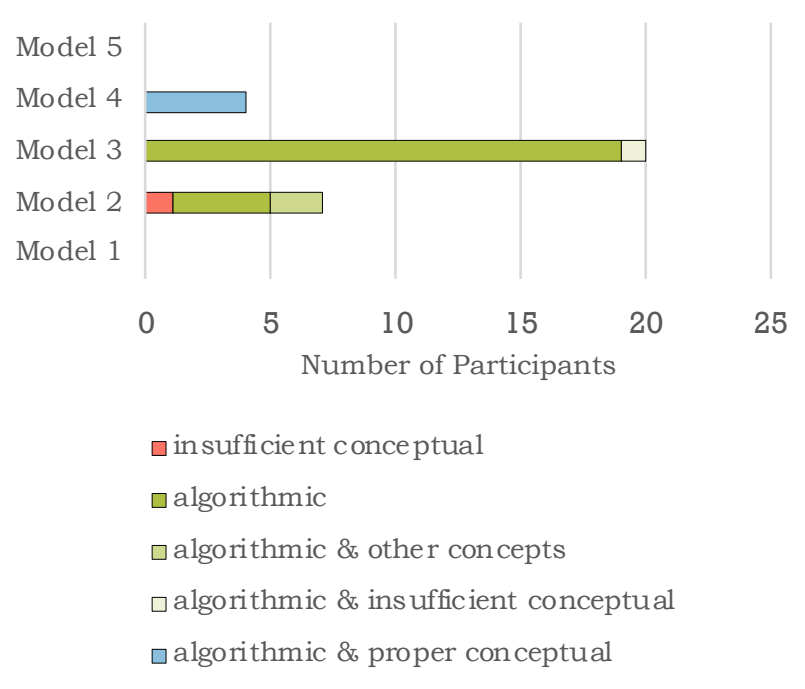

Figure 3. A bar graph illustrating number of participants in each Model and the distribution of strategies used in each Model for Q1. 
Question 3: Approximately what is the internal bond angle of atoms (C-1)-(C-2)-(N-3)?

Like Q1, Q3 could be answered algorithmically, but could also be expanded on with conceptual

knowledge. Again, a majority of participants, 21 (68\%), correctly identified the bond angle as $109.5^{\circ}$,

but unlike Q1, just over half of those participants, 13, did so with proper (algorithmic or conceptual, i.e. non-guessing) reasoning. The most common $(n=15)$ strategy applied was algorithmic, as seen by the child codes in Table 2. In this strategy, participants considered the number of electron groups to identify the geometry and its corresponding angles. Following this method, Cierra, as well as ten others, arrived at the correct answer by recognizing that C-2 is the central atom and it has: "four electron groups which means that is tetrahedral... and with the tetrahedral shape, it comes with a 109.5-degree bond angle." The other four who used this strategy identified the geometry of C-2 but selected the answer of $120^{\circ}$. A second algorithmic strategy that related the hybridization of an atom to a bond angel was employed by only two participants. They both recognized that the internal atom, C-2, is $s p^{3}$-hybridized corresponding to bond angles of $109.5^{\circ}$. Of the participants who used an algorithmic strategy, model placement was, four in Model 2 for arriving at an incorrect answer, eleven in Model 3 for answering correctly without conceptual support, one in Model 3 because their additional conceptual support was insufficient, and one in Model 4 for providing accurate conceptual support (Figure 4) as will be discussed below.

Table 2. A summary of the parent codes and associated child codes observed for $Q 3$, as well as the frequency each child code was observed and number of times it contributed to generating a correct answer and incorrect answer.

\begin{tabular}{ccccc} 
Parent Code & Child Code & $\begin{array}{c}\text { Number of } \\
\text { Times } \\
\text { Observed (n) }\end{array}$ & $\begin{array}{c}\text { Reasoning Led } \\
\text { to Correct } \\
\text { Answer }\end{array}$ & $\begin{array}{c}\text { Reasoning Led } \\
\text { to Incorrect } \\
\text { Answer }\end{array}$ \\
\hline Guessing & Says just guessing/I don't know & 3 & 2 & 1 \\
\hline Deductive & Based on Lewis Structure & 10 & 5 & 5 \\
\hline Algorithmic & $\begin{array}{c}\text { Geometrical reasoning/VSEPR } \\
\text { Based on atom's hybridization }\end{array}$ & $\begin{array}{c}15 \\
2\end{array}$ & $\begin{array}{c}11 \\
2\end{array}$ \\
\hline Conceptual & $\begin{array}{c}\text { Electron groups repel each other } \\
\text { as much as possible }\end{array}$ & 3 & 2 & 0 \\
\hline Other Concepts & Polarity of central atom & 3 & 2 & 1 \\
\hline
\end{tabular}

Of the participants who did not use an algorithm, ten attempted to discern the angle using deductive reasoning based on the Lewis structure. Half of them did so correctly because they either 
ruled out $180^{\circ}$ and correctly guessed between $120^{\circ}$ and $109.5^{\circ}$, or they said it looks like $120^{\circ}$ based off of the picture, but then considered what is surrounding C-2 like Elijah who said:

"Well it's in kind of like a zigzag kind of form and then there's electrons there. So I would think that they have like not completely 180 yeah, not 180, so either between 120 or 109. 120 it's kind of more even too, but I don't think they are because there's a double bond here and uh electrons there, so I would go with 109."

Elijah, along with the nine others who used the given Lewis structure to answer this question were placed in Model 2 on account of their deductive reasoning which is an educated guess based on the explicit information presented and is independent of an algorithm.

Three participants (10\%) attempted to apply prior knowledge. Erika and Harold, invoked polarity, a prior unrelated concept, and were placed in Model 2 as they had no other sound support for their answer. Similarly, Derek considered "charges" that affect the central atom, but he correctly used the VSEPR algorithm which put his response in Model 3.

The three instances of conceptual reasoning relied on considering the repulsion of electrons, but only one participant was able to use it properly. Elijah and Natalia used insufficient conceptual reasoning, by attempting to factor in the repulsion of the lone pair on the adjacent nitrogen, in conjunction with deductive reasoning and were therefore placed in Model 2. Georgia was the only one to be placed in Model 4, as she used proper conceptual reasoning along with the VSEPR algorithmic method. In her discussion, Georgia indicated that: "cause there's four different and so they would be repelled about that much," signifying a deeper understanding of VSEPR theory. Yet, no one provided further explanation in regard to the electron groups repelling each other equal amounts because of the absence of lone pairs of electrons, which is why no one was in Model 5. Finally, only three of the participants resorted to guessing to answer this question and were placed in Model 1.

While a majority of students were able to select the correct answer, the placement of participants into Models, as seen in Figure 4, reflect the low level of reasoning and absence of problem-solving students are required to use. The strategy of trying to discern the bond angle by looking at the given Lewis structure (deductive reasoning), which we deemed to be characteristic of pre-algorithmic reasoning, is why so many participants are in Model 2. Additionally, the ability to answer this question 
Model 5.

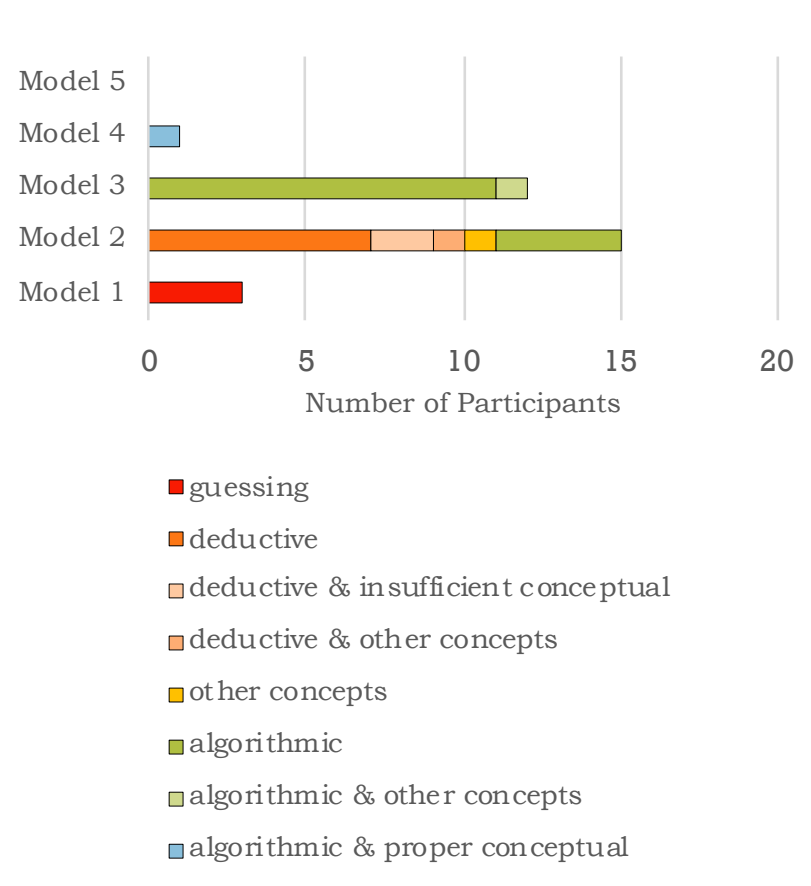

Figure 4. A bar graph illustrating number of participants in each Model and the distribution of strategies used in each Model for Q3.

Question 5: True or False: Rotation about the $\mathrm{C}=\mathrm{C}$ double bond to generate the structure below can occur without breaking any bond.

Categorizing Q5 as algorithmic or conceptual is less straightforward as one could just recall that double bonds do not rotate, but more satisfactorily, one should be able to rationale why they do not rotate using an orbital model. Question 5 elicited a wide variety of explanations for why a double bond can or cannot rotate. Answers were split fairly evenly, with 17 participants claiming that the double bond could rotate while the other 14 said the statement was false. Of those who correctly answered false, upon probing, half simply recalled that double bonds cannot rotate and one provided additional explanation. For consistency with prior questions, fact recall was considered as algorithmic reasoning as it is similar to recalling the VSEPR table. Therefore, those six participants were placed in Model 3. The seventh, Bilal, who was the one participant that provided additional explanation on top of his fact recall, used the analogy "if I had just this one thing [showing a single pen] I could turn and turn and turn it, but if there were two pens there I couldn't" to explain how the double bond is: "restricting its free movement." His ability to supplement fact recall with partial conceptual knowledge, that the 
double bond has two parts, placed his response in Model 4. An additional four participants who answered false were placed in Model 4, for demonstrating a reliance on their conceptual knowledge by mentioning how the double bond has two parts or is above and below which prevents rotation. Gerome was the only participant with a Model 5 response because of his more complete explanation. Opposed to Bilal's analogy, Gerome indicated that he knew the pi bond specifically has: "two layers," and that the p orbitals must be aligned. Finally, two participants who answered false were placed in Model 2 as they applied unrelated concepts, polarity and sterics of the molecule, as the basis of their answer.

The responses became especially interesting when participants answered true. Most used personal conceptions, beliefs that do not appear grounded in theory, to answer the question. It was actually the most common strategy with eleven participants attempting to use their personal knowledge to reason through the question (Table 3). All of them believed that the double bond could rotate based on a comparison of the two Lewis structures - one before and one after rotation - and said they could not identify a reason for any to break. This is likely an example of students only considering the explicit information given as the Lewis structure alone does not suggest the double bond cannot rotate. ${ }^{39}$ Others tried to employ other chemistry knowledge such as identifying steric hindrance that may be involved or the presence of cis and trans isomers. Fatima recalled rotating bonds while learning about Newman projections and thus said that the double bond could rotate. Insufficient conceptual reasoning is responsible for categorizing more than half of the responses for Q5 in Model 2. Finally, two participants just guessed and were placed into Model 1.

Responses to Question 5 showed that when students do not know the proper conceptual explanation to a question, they will attempt to use intuition or other chemistry concepts they deem might be relevant to the question (Figure 5). While it is actually admirable for them to be thinking and weighing multiple concepts, more often a misconception concerning a different concept surfaces. Because of the prevalence of this strategy, 19 participants were in Model 2 or lower. Q5 is more of a conceptual question in regard to understanding why double bonds cannot rotate, which is likely why there was a small increase in population of higher models compared to any other question. 
Table 3. A summary of the parent codes and associated child codes observed for Q5, as well as the frequency each child code was observed and number of times it contributed to generating a correct answer and incorrect answer.

\begin{tabular}{ccccc} 
Parent Code & Child Code & $\begin{array}{c}\text { Number of } \\
\text { Times } \\
\text { Observed (n) }\end{array}$ & $\begin{array}{c}\text { Reasoning Led } \\
\text { to Correct } \\
\text { Answer }\end{array}$ & $\begin{array}{c}\text { Reasoning Led } \\
\text { to Incorrect } \\
\text { Answer }\end{array}$ \\
\hline Guessing & Says just guessing/I don't know & 2 & 0 & 2 \\
\hline Deductive & Personal conception & 11 & 0 & 11 \\
\hline Algorithmic & Fact recall & 7 & 7 & 0 \\
\hline Conceptual & $\begin{array}{c}\text { Because bond is above and below } \\
\text { Double bond has two parts } \\
\text { Pi bond breaks }\end{array}$ & 1 & 4 & 0 \\
\hline Other Concepts & $\begin{array}{c}\text { Because cis and trans isomers } \\
\text { exist }\end{array}$ & 2 & 1 & 0 \\
& $\begin{array}{c}\text { Steric hinderance/degree of } \\
\text { substitution }\end{array}$ & 3 & 1 & 1 \\
& $\begin{array}{c}\text { Newman projections } \\
\text { Polarity of molecule }\end{array}$ & 1 & 1 & 2 \\
& & 1 & 0 & 1 \\
\hline
\end{tabular}
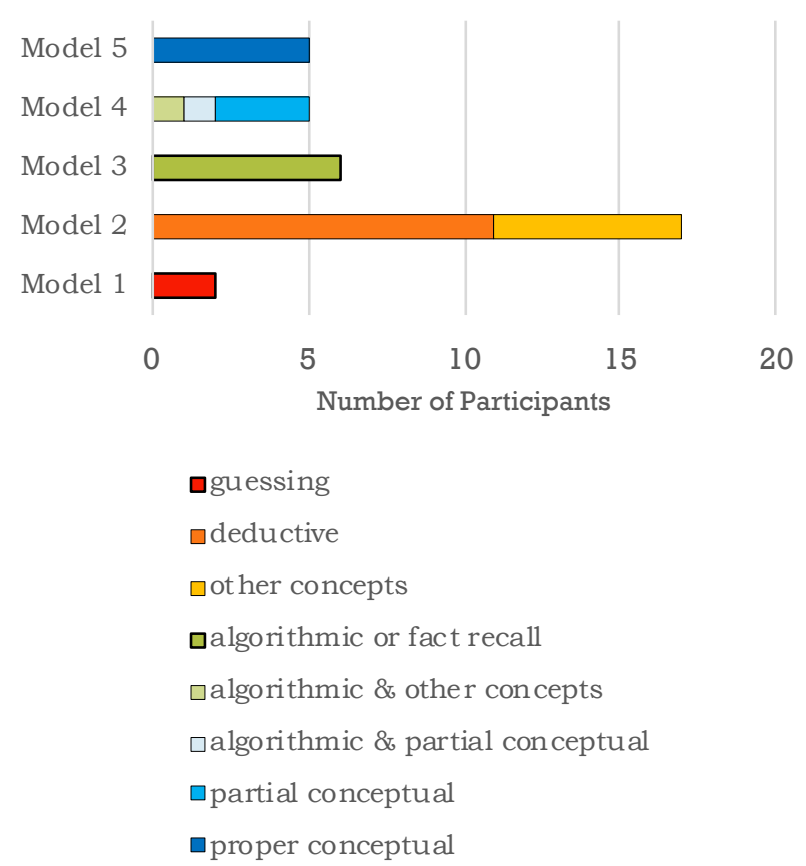

Figure 5. A bar graph illustrating number of participants in each Model and the distribution of strategies used in each Model for Q5.

Question 2: What hybrid orbitals overlap to form the sigma bond between atoms C-1 and C-2?

Aside from Q4, Q2 was the most conceptual question of the questionnaire. Our goal with this question was to see how many students would recognize that two orbitals need to overlap to form a 
bond, but the hybridization of the orbitals does not need to be the same. This proved to be the most challenging, non-drawing question, with only three participants answering correctly. However, we preface the discussion of Q2 with a disclaimer that the wording may have mislead participants to list only one orbital in their answer. Even so though, it is telling that none of the 26 participants (84\%), whose answer was a single orbital, ever questioned how listing only one orbital could give a sufficient answer.

Despite Q2 requiring conceptual understanding to answer, 15 participants still tried to take an algorithmic approach (Table 4) by correlating a sigma bond to a certain hybridized orbital. Interestingly, the most common orbital assigned was a tie between $s p$ and $s p^{3}$ with six each, next was $s p^{2}$ with two, and lastly one participant identified the $s$ orbital as the single bond orbital. This belief that each bond type has a specific orbital is like a recall of a chart so it is considered to be algorithmic reasoning. As none of these algorithmic based answers gave a satisfactory answer, all of these participants were placed into Model 2 (Figure 5).

The difficulty of the question is confirmed by the next most common strategy, guessing. In total, seven participants guessed with no reasoning and were placed into Model 1. A step above guessing, was the one participant, Yasmine, who demonstrated deductive reasoning when she said: "Maybe it would just be the highest between the two. So they both have up to $s p^{2}$, so I'm going to say that." In this case, she chose a commonality between the two atoms to arrive at an educated guess.

Close behind were those who used insufficient conceptual reasoning $(n=3)$ by only considering the hybridization of $\mathrm{C}-1$ and identifying the $s p^{2}$ orbital for sigma bond formation. Although we considered this strategy to be conceptual because participants realized that an orbital from one of the atoms contributes to the bonding, it was considered insufficient conceptual as they failed to recognize that each atom needs to provide an orbital for overlap. Thus, responses were placed in Model 2 because they did not completely answer the question.

The three participants who answered this question correctly considered the hybridizations of C-1 and $\mathrm{C}-2$ and demonstrated an understanding of the concept that two orbitals overlap to form a bond by including a hybrid orbital from each atom in their answer. No responses were placed into Model 3 as the answer cannot be determined algorithmically. Model 4 responses were characterized by just 
listing of the correct two orbitals whereas Model 5 answers identified that the hybrid orbitals of $\mathrm{C} 1$ and C2 must overlap to form the sigma bond. For example, Caleb recognized the hybridization of each carbon then said: "the carbon atoms on both sides of the bonds are hybridized in that way, so when they overlap, they would overlap with those." Interestingly, no one in Model 4 or 5 were confident in their answer unlike some who used an algorithm. Overall, responses to this question confirmed that students prefer algorithmic strategies (Table 4) even when a question requires a conceptual approach.

Table 4. A summary of the parent codes and associated child codes observed for Q2, as well as the frequency each child code was observed and number of times it contributed to generating a correct answer and incorrect answer.

\begin{tabular}{ccccc} 
Parent Code & Child Code & $\begin{array}{c}\text { Number of } \\
\text { Times } \\
\text { Observed (n) }\end{array}$ & $\begin{array}{c}\text { Reasoning Led } \\
\text { to Correct } \\
\text { Answer }\end{array}$ & $\begin{array}{c}\text { Reasoning Led } \\
\text { to Incorrect } \\
\text { Answer }\end{array}$ \\
\hline Guessing & Say just guessing/I don't know & 7 & 0 & 7 \\
\hline Deductive & Try to choose commonality & 1 & 0 & 1 \\
\hline Algorithmic & Each bond type is a specific orbital & 15 & 0 & 15 \\
\hline Conceptual & Two different orbitals overlap & 4 & 3 & 3 \\
\hline $\begin{array}{l}\text { Insufficient } \\
\text { Conceptual }\end{array}$ & Only considers one atom & 3 & 0 & 3 \\
\hline
\end{tabular}

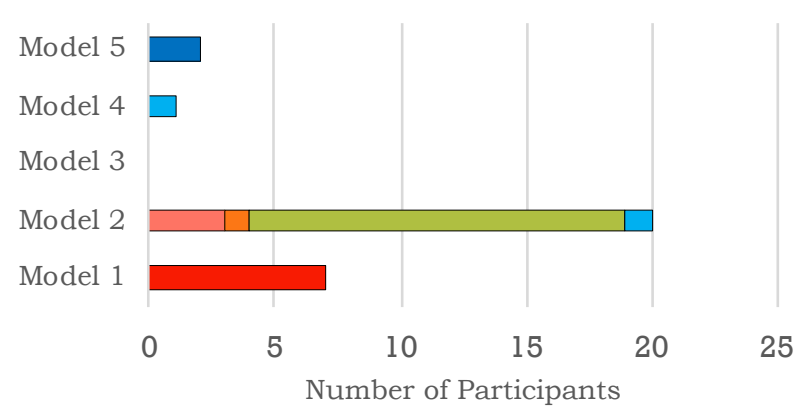

$\begin{array}{ll}\square \text { guessing } & \square \text { insufficient conceptual } \\ \square \text { deductive } & \square \text { algorithmic } \\ \square \text { partial conceptual } & \square \text { proper conceptual }\end{array}$

Figure 5. A bar graph illustrating number of participants in each Model and the distribution of strategies used in each Model for Q2.

Model Population Trends

A summative view of model population by question is given by Figure 7 . As expected, the more algorithmic based questions, Q1 and Q3, have a higher percentage of participants activating a Model 3 (algorithmic) or lower mode of thinking. Very few participants, with correct conceptual understanding, 
felt compelled to give any sort of conceptual support to their answer. A shift to more conceptually based Q5, shows a decrease in Model 3, algorithm use, but only a small increase in Model 4 and Model 5 reasoning levels. Instead, there is still an overwhelming reliance on recall and explicit information provided. Finally, Q2, being conceptually based in nature, is almost completely populated by Model 2 meaning the vast majority of participants could not use conceptual knowledge to answer the question successfully. These trends and observations suggest that students focus on algorithms and recall and are unable to successfully apply conceptual knowledge. As such, there is room in the pedagogy to improve both how material is presented and knowledge assessed.

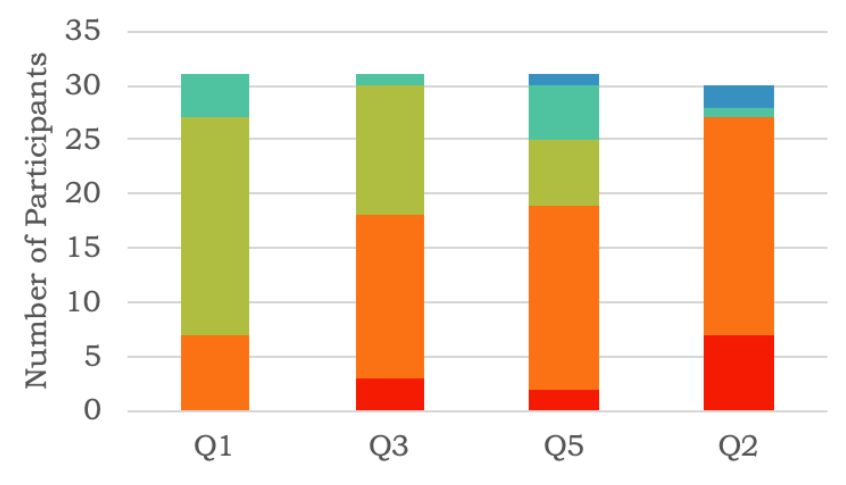

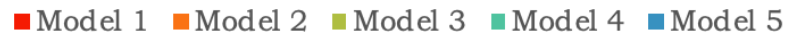

Figure 7. A bar graph illustrating the distribution of models observed for each question, with the questions are ordered from algorithmic to conceptual.

Question 4: On the $C$ below, draw the orbitals for $\mathrm{C}-1$ based on its hybridization and indicate which orbital will be used to make the double bond.

Generation of an acute representation for Q4 requires drawing both hybrid and atomic orbitals. A drawing was considered complete if it had three hybrid orbitals in a trigonal planar arrangement, one $\mathrm{p}$ orbital orthogonal to the hybrid orbitals, and an indication that the $\mathrm{p}$ orbital is used to form the pi bond. The completeness of each portion is summarized in Figure 8.

This question provided insight into how students visualize orbitals within a bonding scheme. As seen in Figure 9, many participants were missing or had only partially complete mental visualizations. In fact, only four participants had some image of all three components while all others were missing at least one of them in their drawing. Two participants did not draw anything after contemplating what they knew. There also appeared to be a disconnect between how participants visualize orbitals within a bonding scheme and their ability to properly describe the components. For example, Bilal drew (Figure 
9a) what one might assume to be an attempt at drawing the $s p^{2}$-hybrid orbitals, but from the transcripts he says: "start off with a s orbitals [draws smallest central dumbbell] and $p$ orbitals I guess [draws larger dumbbell around first one], more $p$ orbital [draws the top oval] Right? Yeah. Okay." revealing that the drawing is actually a combination of inaccurately depicted $s$ and $p$ orbitals. This disconnect between images and what they represent, indicates that while some participants may have a mental image, they may not completely understand it, or vice versa.

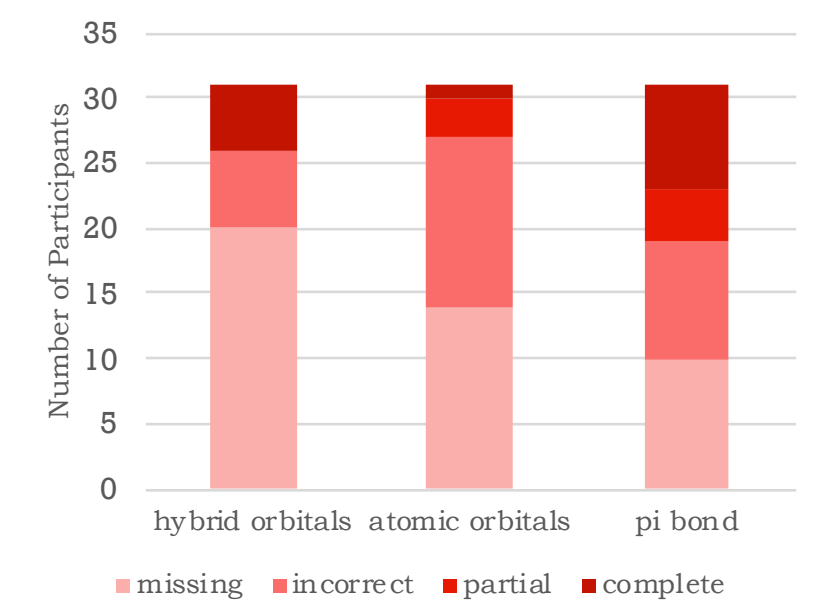

Figure 8. A bar graph showing the distribution of completeness for each part of Q4, namely the hybrid orbitals, atomic orbitals, and pi bond.

This disconnect was most common with the $\mathrm{p}$ orbital and its role in the pi bond. Some remembered the visual of the pi bond forming above and below but could not indicate that it was the p orbital that is involved. Veronica, for example, was able to produce a drawing of the pi overlap (Figure 9b) but had no representation of a p orbital. When drawing, she recalled that the double bond is: "shared along with the axis," and went on to say, "I know like in the pi bond is like the electron could be basically anywhere, so that's why they like draw like that," indicating that she has a mental visualization of the electronics of the pi bond, but not the orbitals involved. Meanwhile, Yasmine (Figure 9c) and Ferris (Figure 9d) both did not have any orbitals in their drawings but still recalled that the p orbital is used to form the double bond. Finally, others could recall that the p orbital forms the pi bond but did not draw it properly. For example, Beatrice (Figure 9e), knew that a p orbital contributed to the pi bond, but drew the p orbital overlapping horizontally with a hybrid orbital. This was particularly surprising as for Q5, Beatrice was placed in Model 4 for recognizing that the pi bond is formed above and below 
the sigma bond, but her mental model does not show why that is the case.
a. Bilal
b. Veronica
c. Yasmine
d. Ferris
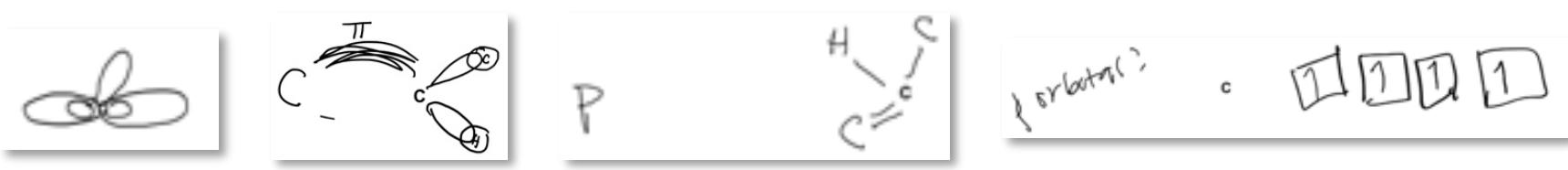

e. Beatrice

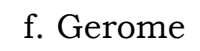

g. Harriet

h. Kelvin

i. Louise
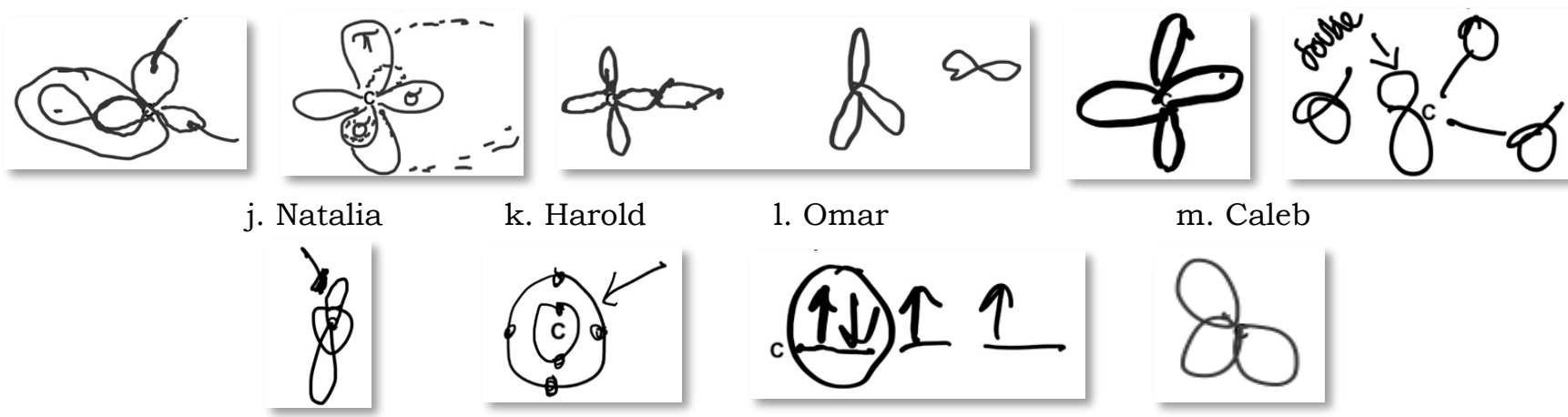

k. Harold

1. Omar

m. Caleb
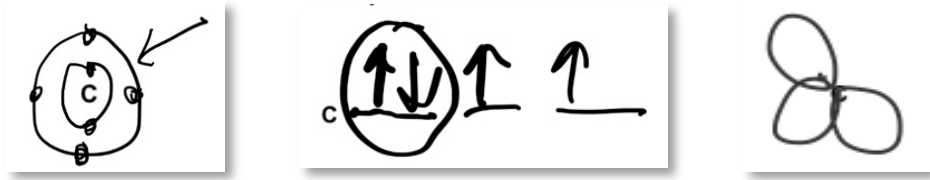

Figure 9. Sample drawings from the questionnaire from a. Bilal, b. Veronica, c. Yasmine, d. Ferris, e. Beatrice, f. Gerome, g. Harriet, h. Kelvin, i. Louise, j. Natalia, k. Harold, 1. Omar, and m. Caleb.

While some could not recall a specific name for the orbitals they drew, several participants, such as Harriet (Figure 9g), specifically remembered that $\mathrm{p}$ orbitals have a dumbbell shape. The dumbbell shape was actually the most common drawing produced either when a participant guessed or only drew what they knew they remembered, p orbitals, all of which resembled an image like Kelvin's (Figure 8h).

The remembrance of $\mathrm{p}$ orbitals was stronger than that of the $s p^{2}$ orbitals as nearly two-thirds of participants were missing hybrid orbitals in their visualizations. Half of these participants only drew atomic orbital(s), either only $p$ orbital(s) or the atomic orbitals that would combine to form the hybrid orbitals. Natalia, for example, drew one s and one p orbital (Figure 9j) based on her incorrect identification of $\mathrm{C}-1$ being $s p$ hybridized in $\mathrm{Q} 1$. The other half of the participants that were missing hybrid orbitals either drew lines to represent the bonding as in a Lewis structure, or atomic structure representations. For instance, Harold drew a Bohr diagram (Figure 9k) and Omar used arrows to represent electrons (Figure 91). These misconceptions have been observed before ${ }^{15}$ and are examples of students using previously learned conceptual frameworks. Taber coined this phenomenon as a "pedagogical teaching impediment" as it is course instruction rather than everyday experiences that 
have led to the misconception. ${ }^{18}$ Also, since these responses are actually models to describe atoms these examples could be considered cases of model confusion. ${ }^{40}$

On their own, the drawings and descriptions given by participants has been telling but what is equally interesting is comparing the quality of drawing to the responses given to the conceptually based questions. Beginning with the more conceptually based question, Q2, there were only five participants who had a complete accurate drawing of hybrid orbitals, however, four of these participants were placed in Model 2 for Q2 and only one was in Model 5. This suggests that reproduction of an orbital drawing does not imply a student knows how it is used in bonding. Another way to study the relationship between hybrid orbital drawing and Q2 is to examine the drawings of students who were placed in a high model for Q2. As stated earlier, one participant, Georgia, was in Model 5 and had a complete hybrid orbital drawing. There was one other participant, Caleb, who was placed in Model 5, but, in stark contrast, after reading the prompt for Q4 he said "Hmm. I forget this. I don't know how to draw this, honestly. I don't know. Okay. There you go." When probed further about what the generic drawing produced represented (Figure 9m) he said "I know that these are like the, what is it? The electron clouds or whatever, where they're supposed to be like remotely." The last participant in a higher model, Model 4, Veronica, (Figure 9b) had incorrect hybrid orbitals as she neglected to show the orbital that would be used to form the sigma bond with C-2. Overall, we found no correlation between completeness and accuracy of drawings of hybrid orbitals and quality of answers to Q2.

A goal of Q5 was to see how many participants would recognize that the pi bond is above and below the sigma bond. A natural inference would be that these students also have an accurate mental representation of a $p$ orbital. For Q5, five participants were placed in Model 4 and one, Gerome, was placed in Model 5. Gerome had a complete and accurate depiction of both the $p$ orbital and the pi bond. Surprisingly though, none of the five participants in Model 4 generated an accurate depiction of the $p$ orbital and three of them made no indication for how the pi bond is formed. Three of these participants, Beatrice, Natalia, and Bilal were discussed previously. Of the remaining two, Cierra, similarly to Bilal, drew multiple $p$ orbitals where normally hybrid orbitals are placed and did not identify what is used to make the pi bond. Finally, Aya drew nothing and in trying to identify which 
orbital would form the double bond stated, "The only thing I feel like I remember is that the double bonds, I feel like those are related to the pi bonds." This finding shows that even students with what on the surface appears to be conceptual understanding still struggle to picture and or have a mental visualization of orbitals. In looking the other direction, those who had accurate drawings of the $p$ orbitals and how they did on Q5, there were only two participants, not discussed previously, who had partial $p$ orbital drawings and both were placed in Model 2 for Q5. We postulate that one explanation of these findings is the cognitive overload that can occur when students attempt to simultaneously consider both the symbolic and sub-microscopic domains of Johnstone's triangle. ${ }^{41}$ These findings also support that the modality of a question also plays a role in how well students can demonstrate understanding. ${ }^{35}$

\section{CONCLUSION}

When examining how the participants approach hybridization questions, it became apparent that they succeed and are most confident on the questions that can be solved using an algorithm such as a table or simple "if this, then that" thought process. Specifically, Q1 was the most correctly answered question as 24 of the 31 participants correctly identified the hybridization of C-1 and C-2 using an algorithmic strategy. When a participant could not recall an algorithm, they frequently attempted to apply other chemistry concepts or use deductive reasoning, based on explicit information or personal conceptions, as seen for Q3 and Q5. Moving to conceptually based questions, only a small increase in population of higher models, that include accurate conceptual reasoning, was found (Figure 7).

Overall, this points to students' reliance on algorithms to give a correct answer, the endpoint, without the need for conceptual reasoning.

Regarding conceptual reasoning, Q5, considering the ability to rotate a pi bond, had the highest number of students with partial or proper conceptual reasoning. Additionally, about half of the participants correctly answered that the pi bond cannot rotate without breaking. In contrast, understanding of sigma bonding, Q2, is low as evidenced by only three participants answering correctly, with low confidence, and a majority of participants being placed in Model 2.

Correspondingly, in $\mathrm{Q} 4$, over half of the participants could attempt to draw an atomic orbital whereas 
only one-third could give a representation of hybrid orbitals. Taken together, students appear to have experienced a greater emphasis on learning $p$ orbitals and pi bonding.

In comparing the quality of drawings from Q4 with a participant's model placement for Q5, a lack in connection between symbolic and microscopic domains was found. None of the participants who were placed in Model 4 for Q5 had an accurate depiction of the $p$ orbital and over half of them could not identify how the pi bond was formed. Likewise, all those who had at a partially correct $p$ orbital drawing for Q4, said in Q5 that a pi bond can rotate without breaking. Overall, the completeness of drawing in Q4 was not a good predictor of a student's ability to answer a question using conceptual knowledge and vice versa. One explanation for this observation could be the different modalities, drawing versus verbal explanation, of the question. Ryan and Stieff have shown that assumptions about student understanding of how a feature relates to a model cannot be made by the information collected from the student in only one modality. ${ }^{35}$ We certainly saw this was the case concerning pi bonding and a need to help connect the two modalities to ensure full understanding rather than partial recall. In fact, this is one of the reasons we split conceptual reasoning between Model 4 and Model 5, to separate those who could recall the concept and its fundamentals versus those who could apply the concept to give an answer with strong explanatory power.

Overall, these findings suggest that current pedagogy places an emphasis on pi bonding but lost to students is a mental image of the overlap of orbitals to form bonds. Aya, the one participant who left Q2 blank, reiterated this through her thoughts as she tried to visualize and contemplate what she knew about sigma bonds before ultimately moving onto the next: "I remember the, the visual in the text and in the lecture of the um, bonds on like a, I feel like pi bonds, they're more stable cause they had like two shared orbitals, one below and one above or maybe that's sigma, like I remember that the sort of visual of having the things above." Ultimately, Aya did not feel comfortable putting an answer for this question, but it was interesting that she was able to visualize pi bonds, but not sigma bonds. Implications for Teaching

As hybridization theory is an abstract concept, it poses a challenge for teaching as the terms used to describe the model (a hybrid orbital, $s p^{3}$ orbital, sigma bonds) are not meaningful until the overall concept is understood. ${ }^{18}$ Commonly, for abstract topics, definitions are initially learned through rote 
memorization. Incidentally, we noticed an inability of many participants to use the terms properly, for example, $p$ orbital was commonly referred to as the pi orbital, suggesting limited integration. A corollary to definitions for the topic at hand are the algorithms students are taught to identify the hybridization of an atom and the molecular geometry. To be clear, these algorithms are useful and have their place as a starting point for learning hybridization theory. However, from this study, we have found that for a majority of students, mastering the ability to use the algorithm is an end point, leaving out the need for a conceptual understanding of the topic.

In part, we ourselves, the instructors and standardized test curators, may have played a role in this belief held by students, by using mainly algorithmic based questions on assessment items. ${ }^{27}$ During our study, we did see a small increase in conceptual reasoning as the question shifted toward conceptual basis. Therefore, we recommend having a variety of algorithmic and conceptually based questions. Variety is key. Central to the theoretical framework for this study, as understanding of a theory increased the explanatory power, where the power comes from conceptual reasoning, of an answer increases. If algorithmic reasoning is viewed as a starting point, then it should be assessed to determine where on the continuum, and therefore the extent of their conceptual understanding, students' reasoning lies. In fact, during this study we identified a number of students who for Q1 and Q3 (algorithmically based) were struggling or unable to even use the algorithm. This also provides a means to identify which students may need additional help. However, if the goal of using algorithms is to pave the path for conceptual understanding, then it is also necessary to include conceptually based questions, as otherwise students do not feel the need to demonstrate their conceptual knowledge.

Interestingly, the bar graphs of the Model population for each question, Figures 2-6, resemble bellcurves that are often seen in grade distributions. For algorithmic based questions, Q1, the curve is tight with a high population in Model 3, correct algorithmic reasoning. As the conceptual basis of the question increases though, the curve gets wider, Q3 and Q5, and eventually shifts to a higher population in Model 2. The high population in Model 2 indicates students' inability to properly apply conceptual knowledge to answer the questions. We also interpret this as adding validity to our theoretical framework that student understanding can be measured by looking at their ability to apply conceptual knowledge. Unfortunately, this level of assessment is currently impractical due to the time 
intensive nature of both developing an answer (exams are typically a short time period) and assessment. However, through advances in technology, such as lexical analysis and digital interactives, this kind of assessment may become feasible.

In asking conceptual questions though, it is important to assess understanding via multiple modalities. ${ }^{35}$ Especially in comparing answers to Q5 and drawings in Q4, it was evident that some participants were better able to discuss pi bonding verbally while others were better able to use images. Additionally, this may be a way to distinguish the top-level students as those with the most complete understanding should be comfortable using multiple modalities. Therefore, asking only one modality may erroneously lead an instructor to think a student has a complete conceptual understanding or no conceptual understanding. ${ }^{35}$

From our interviews, only one participant was able to give a high-level answer for Q5 (Model 5) and provide a quality drawing in Q4. This strongly suggest the need for improved pedagogy that connects the visualizations of orbitals (symbolic domain) with its implications in bonding (sub-microscopic domain). ${ }^{41}$ In general, students struggled to draw orbitals, which is understandable as they are trying to depict three-dimensional objects, that they likely do not fully understand, in a two-dimensional representation. This limits their ability to interact with and manipulate the models of hybridization. Additionally, from Taber we know students: "can only interpret such information in terms of our existing conceptual schemes, which provide the contextual frameworks for converting the utterances of teachers and text from books into meaningful mental images."18 Therefore, we believe we have identified a need to provide students with more opportunities to manipulate orbitals, for instruction to spend more time discussing and connecting orbital models with conceptual theories, in particular orbital overlap to form bonds, and to include orbital representations on assessment items. However, drawing orbitals appears to place a high cognitive demand on students, so it would be better to use automatically generated orbitals which is possible through the use of technology.

\section{ASSOCIATED CONTENT}

The Supporting Information is available on the ACS Publications website at DOI: 10.1021/acs.jchemed.XXXXXXX. 
The questionnaire used in the interviews, rationales used during questionnaire development, working definitions of algorithmic and conceptual reasoning, and the coding systems used (PDF, DOCX)

\section{AUTHOR INFORMATION}

Corresponding Author

*E-mail: sarah@alchem.ie

ORCID

Sarah E. Wegwerth: 0000-0003-3303-0081

\section{ACKNOWLEDGMENTS}

We thank the participants for both their time and thoughts during the interviews. We also thank the instructors who distributed the recruitment emails, Michelle Driessen and Matthew Mio, Ginger Shultz for guidance on research strategies and best practices, Elizabeth Gross for designing the graphical abstract, and Typhany Jones for research support. Funding for this project was provided by an NSF SBIR Grant \#1659983.

\section{REFERENCES}

(1) Grushow, A. Is It Time to Retire the Hybrid Atomic Orbital? J. Chem. Educ. 2011, 88 (7), 860862.

675 (2) Tro, N. J. Retire the Hybrid Atomic Orbital? Not so Fast. J. Chem. Educ. 2012,89 (5), $567-568$.

(3) DeKock, R. L.; Strikwerda, J. R. Retire Hybrid Atomic Orbitals? J. Chem. Educ. 2012, 89 (5), 569.

(4) Landis, C. R.; Weinhold, F. Comments on “Is It Time to Retire the Hybrid Atomic Orbital?" J. Chem. Educ. 2012, 89 (5), 570-572.

(5) Truhlar, D. G. Are Molecular Orbitals Delocalized? J. Chem. Educ. 2012, 89 (5), 573-574.

(6) Hiberty, P. C.; Volatron, F.; Shaik, S. In Defense of the Hybrid Atomic Orbitals. J. Chem. Educ. 2012, 89 (5), 575-577.

(7) Chang, R.; Overby, J. Chemistry, 13th ed.; McGraw Hill: New York, NY, 2018.

(8) Ebbing, D. D.; Gammon, S. D. General Chemistry, 11th ed.; Cengage: Boston, MA, 2017.

(9) Jespersen, N. D.; Brady, J. E.; Hyslop, A. Chemistry: The Molecular Nature of Matter, 7th ed.; Wiley: Hoboken, NJ, 2014.

(10) Tro, N. J. Chemistry: A Molecular Approach, 5th ed.; Pearson: New York, NY, 2020.

(11) Brown, W. H.; Iverson, B. L.; Anslyn, E.; Foote, C. S. Organic Chemistry, 8th ed.; Cengage: Boston, MA, 2018.

(12) Klein, D. R. Organic Chemistry, 3rd ed.; Wiley: Hoboken, NJ, 2017. 2018. 
(14) Wade, L. G.; Simek, J. W. Organic Chemistry, 9th ed.; Pearson: New York, NY, 2017.

(15) Salaha, H.; Dumonb, A. Conceptual Integration of Hybridization by Algerian Students Intending to Teach Physical Sciences. Chem. Educ. Res. Pract. 2011, 12 (4), 443-453.

(16) Nakiboglu, C. Instructional Misconceptions of Turkish Prospective Chemistry Teachers About Atomic Orbitals and Hybridization. Chem. Educ. Res. Pr. 2003, 4 (2), 171-188.

(17) Zoller, U. Students' Misunderstandings and Misconceptions in College Freshman Chemistry (General and Organic). J. Res. Sci. Teach. 1990, 27 (10), 1053-1065.

(18) Taber, K. S. Building the Structural Concepts of Chemistry: Some Considerations From Educational Research. Chem. Educ. Res. Pr. 2001, 2 (2), 123-158.

(19) Birk, J. P.; Kurtz, M. J. Effect of Experience on Retention and Elimination of Misconceptions about Molecular Structure and Bonding. J. Chem. Educ. 1999, 76 (1), 124-128.

(20) Çalıs, S. An Examination of the Achievement Levels of Acquisitions in Hybridization: High School Sample. Univers. J. Educ. Res. 2018, 6 (8), 1659-1666.

(21) Cooper, M. M.; Underwood, S. M.; Hilley, C. Z.; Klymkowsky, M. W. Development and Assessment of a Molecular Structure and Properties Learning Progression. J. Chem. Educ. 2012, 89 (11), 1351-1357.

(22) Bodner, G. M. Constructivism: A Theory of Knowledge. J. Chem. Educ. 1986, 63 (10), 873.

(23) Domin, D.; Bodner, G. Using Students' Representations Constructed during Problem Solving to Infer Conceptual Understanding. J. Chem. Educ. 2012, 89 (7), 837-843.

(24) Bruner, J. The Process of Education, Revised.; Harvard University Press: Cambridge, 1977.

(25) Nurrenbem, S. C.; Pickering, M. Concept Learning versus Problem Solving: Is There a Difference? J. Chem. Educ. 1987, 64 (6), 508-510.

(26) Nakhleh, M. B.; Mitchell, R. C. Concept Learning versus Problem Solving: There Is a Difference. J. Chem. Educ. 1993, 70 (3), 190-192.

(27) Stowe, R. L.; Cooper, M. M. Practicing What We Preach: Assessing "Critical Thinking" in Organic Chemistry. J. Chem. Educ. 2017, 94 (12), 1852-1859.

(28) Grove, N. P.; Cooper, M. M.; Rush, K. M. Decorating with Arrows: Toward the Development of Representational Competence in Organic Chemistry. J. Chem. Educ. 2012, 89 (7), 844-849.

(29) Cooper, M. M.; Corley, L. M.; Underwood, S. M. An Investigation of College Chemistry Students' Understanding of Structure-Property Relationships. J. Res. Sci. Teach. 2013, 50 (6), 699-721.

(30) Bhattacharyya, G.; Bodner, G. M. "It Gets Me to the Product": How Students Propose Organic Mechanisms. J. Chem. Educ. 2005, 82 (9), 1402.

(31) Niaz, M. Progressive Transitions from Algorithmic to Conceptual Understanding in Student Ability to Solve Chemistry Problems: A Lakatosian Interpretation. Sci. Educ. 1995, 79 (1), 19-36.

(32) de Cataldo, R.; Griffith, K. M.; Fogarty, K. H. Hands-On Hybridization: 3D-Printed Models of Hybrid Orbitals. J. Chem. Educ. 2018, 95 (9), 1601-1606. 
(33) Penny, M. R.; Cao, Z. J.; Patel, B.; Sil dos Santos, B.; Asquith, C. R. M.; Szulc, B. R.; Rao, Z. X.; Muwaffak, Z.; Malkinson, J. P.; Hilton, S. T. Three-Dimensional Printing of a Scalable Molecular Model and Orbital Kit for Organic Chemistry Teaching and Learning. J. Chem. Educ. 2017, 94 (9), 1265-1271.

(34) Bretz, S. L. Qualitative Research Designs in Chemistry Education Research. In Nuts and Bolts of Chemical Education Research; ACS Symposium Series; American Chemical Society, 2008; Vol. 976, pp 79-99.

(35) Ryan, S. A. C.; Stieff, M. Drawing for Assessing Learning Outcomes in Chemistry. J. Chem. Educ. 2019, 96 (9), 1813-1820.

(36) Creswell, J. W. Educational Research Planning, Conducting, and Evaluating Quantitative and Qualitative Research, Fourth.; Pearson: Boston, 2012.

(37) Preparing for Your ACS Examination in Organic Chemistry The Official Guide; American Chemical Society, Division of Chemical Education, Examinations Instiute: Milwaukee, 2002.

(38) McHugh, M. L. Lessons in Biostatistics Interrater Reliability: The Kappa Statistic. Biochem. Medica 2012, 22 (3), 276-282.

(39) Cooper, M. M.; Grove, N.; Underwood, S. M.; Klymkowsky, M. W. Lost in Lewis Structures: An Investigation of Student Difficulties in Developing Representational Competence. J. Chem. Educ. 2010, 87 (8), 869-874.

(40) Carr, M. Model Confusion in Chemistry. Res. Sci. Educ. 1984, 14 (1), 97-103.

(41) Johnstone, A. H. Why Is Science Difficult to Learn? Things Are Seldom What They Seem. J. Comput. Assist. Learn. 1991, 7 (2), 75-83. 